\title{
SUSY Higgs Searches at DØ, Tevatron
}

\author{
Prolay Kumar Mal \\ (for DØ Collaboration) \\ University of Notre Dame, \\ Notre Dame, Indiana 46556, USA
}

\begin{abstract}
During Run II of the Tevatron collider, D $\varnothing$ collaboration has made extensive searches for the neutral MSSM Higgs bosons $(\phi)$, produced in p p collisions at $\sqrt{\mathrm{s}}=1.96 \mathrm{TeV}$. Two such analyses, addressing inclusive $\phi$ production with $\phi \rightarrow \tau^{+} \tau^{-}$, and associated $\phi b(\bar{b})$ production with $\phi \rightarrow b \bar{b}$ are reported here. No excess of events above the background expectation has been observed in any of these analyses. The results are combined to set constraints on the MSSM parameter space.
\end{abstract}

Keywords: MSSM, Higgs, DØ, Tevatron

PACS: $11.30 . \mathrm{Pb}, 12.60 . \mathrm{Fr}, 13.85 . \mathrm{Rm}, 14.80 . \mathrm{Cp}$

\section{INTRODUCTION}

The Standard Model (SM) of particle physics has been observed to be a consistent theory of fundamental particles and their interactions up to the energies they have been studied although one remaining essential ingredient the Higgs boson is yet to be discovered. Theoretical considerations teach us that SM can not be the ultimate theory of elementary particles and their interactions. Amongst many other compelling theories, the Minimal Supersymmetric extension of the SM (MSSM) is one of the viable theoretical frameworks which has the potential to overcome some of the short comings of the SM by incorporating minimal supersymmetric particle spectrum.

Because of its supersymmetric structure, the MSSM[1][2] requires at least two Higgs doublets to generate masses to both "up"-type and "down"-type quarks (and the respective charged leptons). Such a two-Higgs-doublet model predicts five physical Higgs bosons: two CP-even Higgs bosons, $\mathrm{h}$ and $\mathrm{H}$, one $\mathrm{CP}$-odd Higgs bosons, A and a pair of charged Higgs bosons, $\mathrm{H}^{ \pm}$. The MSSM Higgs sector at the lowest order can fully be described in terms of one Higgs boson mass $\left(\mathrm{M}_{\mathrm{A}}\right.$ is chosen in CP-conserving scenario and $\mathrm{M}_{\mathrm{H}^{ \pm}}$in CP-violating scenario) and $\tan \beta=\mathrm{v}_{2} / \mathrm{v}_{1}$, where $\mathrm{v}_{2}\left(\mathrm{v}_{1}\right)$ refers to the Higgs field that couples to the "up"(“down")-type quarks. However, additional parameters viz., $\mathrm{M}_{\mathrm{SUSY}}, \mathrm{M}_{2}, \mu$ and $\mathrm{m}_{\tilde{\mathrm{g}}}$ enter at the level of radiative corrections. It is to be noted that the coupling of the Higgs boson A to "down"-type quark such as bottom quark is proportional to $\tan \beta$. So with respect to the $\mathrm{SM}$, the production cross section for $\mathrm{A}$ in association with bottom quark(s) gets enhanced by a factor of $\tan ^{2} \beta$. Furthermore, at large $\tan \beta(\approx 50)$, there is mass degeneracy between the Higgs bosons $\mathrm{A}$ and $\mathrm{h}$ or $\mathrm{H}$ depending on the value of $\mathrm{m}_{\mathrm{A}}$. Therefore, the total production cross section for MSSM neutral Higgs bosons i.e., $\mathrm{h} / \mathrm{H} / \mathrm{A} \equiv \phi$ is twice that of the Higgs boson A. Previously, LEP experiments have set the mass of light Higgs boson $m_{h}$ to be greater than 92.8 $\mathrm{GeV}[3]$ at $95 \% \mathrm{CL}$. 
The MSSM neutral Higgs bosons $(\phi)$ mostly decay into $b \bar{b}$ pairs $(90 \%)$ or into $\tau^{+} \tau^{-}$pairs $(\sim 8 \%)$. So, at the Tevatron $p \bar{p} \rightarrow \phi(\rightarrow b \bar{b}) b(\bar{b}) X$ and $p \bar{p} \rightarrow \phi\left(\rightarrow \tau^{+} \tau^{-}\right) X$ processes are considered to be the most promising channels to look for the signature of MSSM neutral Higgs bosons. Here we report on the searches for MSSM neutral Higgs bosons in the above channels performed by the DØ experiment [4]. The combined results are interpreted in different MSSM benchmark scenarios[5]: " $\mathrm{m}_{\mathrm{h}}^{\max }$ " and "no-mixing".

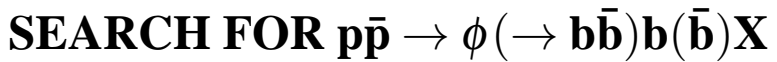

The analysis focuses on MSSM neutral Higgs boson production in association with one or two bottom quark(s) resulting in three or four bottom quarks in the final state while, $\mathrm{CP}$-conservation in the Higgs sector is assumed. $260 \mathrm{pb}^{-1}$ of $\mathrm{D} \varnothing$ data collected between November 2002 and June 2004 have been utilized for this analysis. The data are first filtered using a dedicated on line trigger designed to maximize the signal acceptance. The Secondary Vertex (SV) tagging algorithm has been used to select the b-jets. The DØ SV b-tagging algorithm selects central b-jets $\left(\mathrm{p}_{\mathrm{T}}>35 \mathrm{GeV}\right)$ with an efficiency of $\approx 55 \%$ while the mis-tag rate for similar light quark jets is about $1 \%$.

For Higgs masses between 90 and $150 \mathrm{GeV}$, the $h b \rightarrow b \bar{b} b$ signal events have been generated using the Pythia [6] event generator followed by the full D $\varnothing$ detector simulation and reconstruction. The Pythia generated $\mathrm{p}_{\mathrm{T}}$ and rapidity spectra of the Higgs bosons have been adjusted to those from NLO calculations [7]. The largest background contribution from QCD multijet processes have been determined directly from data while contributions from other background processes such as $\mathrm{t} \overline{\mathrm{t}}, Z^{0}(\rightarrow b \bar{b})+\mathrm{jets}$ have been determined from Monte Carlo.

The selected events are required to have at least three b-tagged jets (within $|\eta|<$ 2.5) with $\mathrm{p}_{\mathrm{T}}>35,20$ and $15 \mathrm{GeV}$ respectively for the leading $\mathrm{p}_{\mathrm{T}}$ jets. The invariant mass distribution of the two leading $\mathrm{p}_{\mathrm{T}}$ jets in data is then compared with that of the background expectation and no excess has been observed. Fig. 1(a) shows the limit on the production cross section at $95 \% \mathrm{CL}$, as a function of $\mathrm{m}_{\mathrm{A}}$ for $\tan \beta=80$ in "no mixing" scenarios. The results are also interpreted in the "maximal mixing" case and the limits in $\tan \beta-\mathrm{m}_{\mathrm{A}}$ plane for both scenarios are shown in Fig. 1 b).

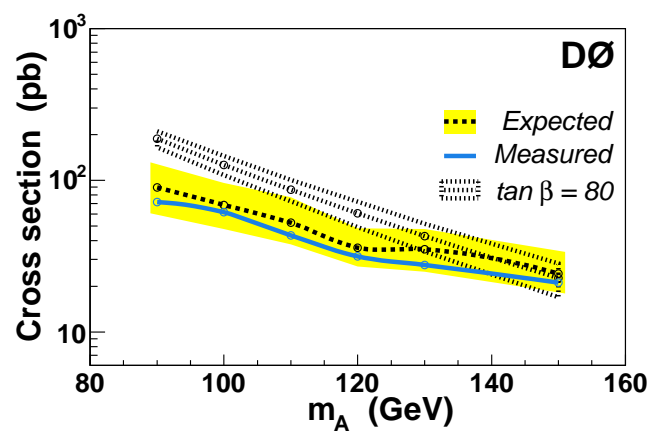

(a)

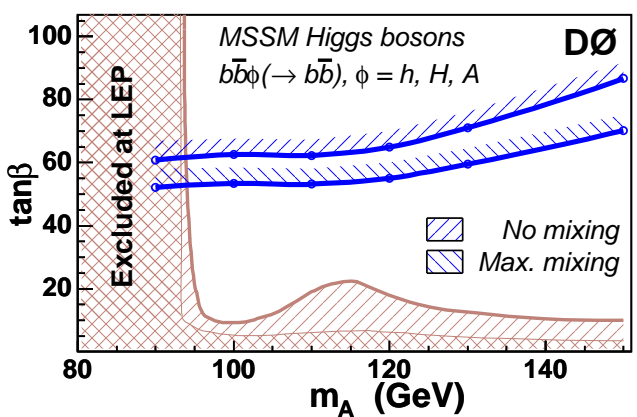

(b)

FIGURE 1. (a) The upper limits on the production cross section as a function of Higgs mass and (b) the limits on $\tan \beta-\mathrm{m}_{\mathrm{A}}$ plane at $95 \% \mathrm{CL}$. 


\section{SEARCH FOR $\mathbf{p} \overline{\mathbf{p}} \rightarrow \phi\left(\rightarrow \tau^{+} \tau^{-}\right) \mathbf{X}$}

The analysis is based on $325 \mathrm{pb}^{-1}$ of data collected by the $\mathrm{D} \varnothing$ experiment during the period September 2002 to August 2004. The signal consists of a pair of tau leptons. One of the tau leptons is required to decay leptonically into electron or muon, leading to three final state signature: $e \tau_{h}, \mu \tau_{h}$ and $e \mu$, where $\tau_{h}$ represents a hadronically decayed tau lepton. The three types of hadronically decayed $\tau$ leptons,

- $\tau$-type 1: a single track with a calorimeter cluster without any electromagnetic sub clusters (1-prong, $\pi$-like)

- $\tau$-type 2: a single track associated with a calorimeter cluster and electromagnetic sub clusters (1-prong, $\rho$-like)

- $\tau$-type 3: two or at least three tracks with invariant mass below 1.1 or $1.7 \mathrm{GeV}$ respectively (3-prong)

have been utilized in this analysis. A set of neural networks ${ }^{\dagger}$, one for each type have been used for further discrimination over the backgrounds.

The signal as well as various background processes have been generated using Pythia. Events are then passed through the full chain of $\mathrm{D} \varnothing$ detector simulation and reconstruction software. Apart from the QCD multijet background where a jet mimics an electron/muon from tau lepton, all other background processes are normalized to NLO and NNLO $\left(Z^{0}\right.$ boson, $W^{ \pm}$boson, Drell-Yan and di-boson) cross sections.

The reconstructed visible mass defined as $\mathrm{M}_{\mathrm{vis}}=\sqrt{\left(\mathrm{P}_{\tau 1}+\mathrm{P}_{\tau 2}+\mathbf{P}_{\mathrm{T}}\right)^{2}}$, where $\mathrm{P}_{\tau 1,2}$ is the four vector of the visible tau decay products and $\mathbb{P}_{\mathrm{T}}=\left(\mathbb{E}_{\mathrm{T}}, \mathbb{E}_{\mathrm{X}}, \mathbb{E}_{\mathrm{y}}, 0\right)$, is used to discriminate between signal and background processes. No excess over the background expectation has been observed. The Figs. 2(a)-(b) show the distributions of $\mathbf{M}_{\text {vis }}$ (denoted as "Inv. Mass $\left(\mathrm{e} / \mu, \operatorname{tau}_{\mathrm{had}}, \mathbb{E}_{\mathrm{T}}\right)$ " and "Inv. Mass $\left(\mathrm{e}, \mu, \mathbb{E}_{\mathrm{T}}\right)$ " respectively in the figure) for different final states, while Fig. 2(c) displays the upper limits on production cross section as a function of Higgs mass.

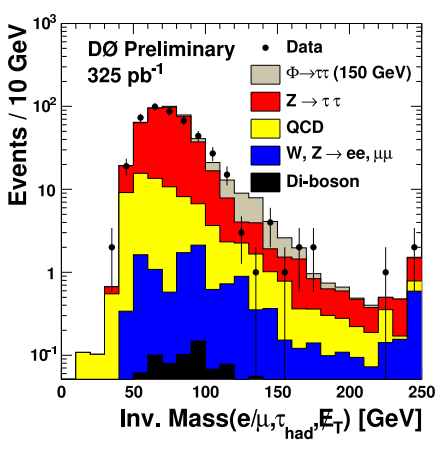

(a)

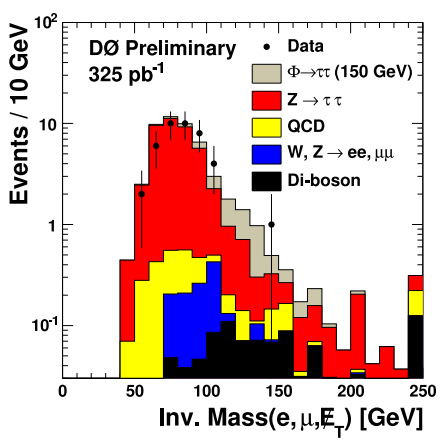

(b)

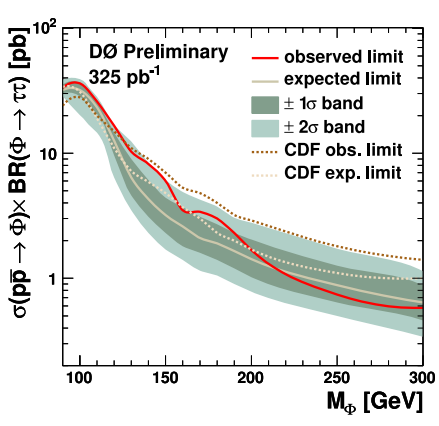

(c)

FIGURE 2. The distribution of reconstructed visible mass $\mathrm{M}_{\mathrm{vis}}$ (see text) for (a) $e \tau_{h}+\mu \tau_{h}$ channel, and (b) $e \mu$ channel. (c) The upper limits on the production cross section as a function of Higgs mass at $95 \%$ CL.

\footnotetext{
$\dagger$ Identical to the ones used for $Z^{0} / \gamma^{*} \rightarrow \tau^{+} \tau^{-}$cross section measurements [8]
} 


\section{COMBINED LIMITS}

The DØ results obtained from the above two analyses [9] [10] are combined together and are re-interpreted in " $\mathrm{m}_{\mathrm{h}}^{\max }$ " and "no-mixing" scenarios. Fig. 3 shows the current DØ limits on MSSM parameter space. The similar limits obtained by the CDF [11] are also displayed for the purpose of comparison.

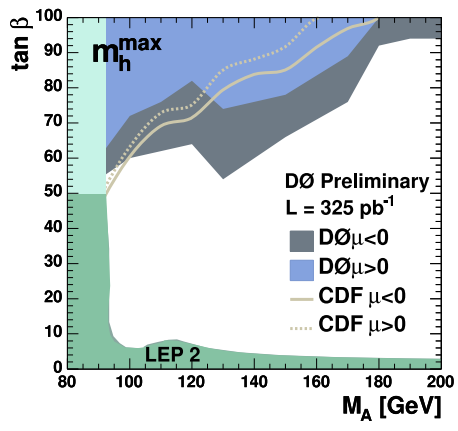

(a)

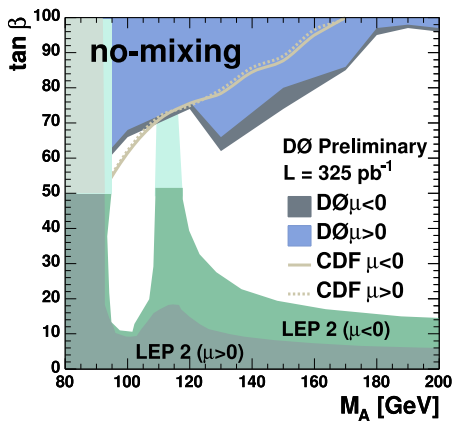

(b)

FIGURE 3. The excluded region in the $\left(\mathrm{M}_{\mathrm{A}}, \tan \beta\right)$ plane for the $\mathrm{m}_{\mathrm{h}}^{\max }$ and no-mixing scenario with $\mu= \pm 200 \mathrm{GeV}$.

\section{CONCLUSIONS}

DØ has performed searches for the MSSM neutral Higgs bosons and the current D $\varnothing$ limit on MSSM parameter space is the most sensitive to date. Additional search channels like $p \bar{p} \rightarrow \phi\left(\rightarrow \tau^{+} \tau^{-}\right) b(\bar{b}) X$, advanced analysis techniques and larger dataset will provide further scope to improve the sensitivity in future.

\section{REFERENCES}

1. H. P. Nilles, Phys. Rep., 110, 1 (1984).

2. H. P. Nilles and H. E. Haber and G. L. Kane, Phys. Rep., 117, 75 (1985).

3. The LEP Working Group for Higgs Boson Searches, CERN-PH-EP/2006-001 (2006).

4. V. M. Abazov et al. (DØ Collaboration), Nucl. Instrum. and Methods A 565, 463 (2006).

5. M. Carena et al, Eur.Phys.J. C, 45, 797 (2006).

6. T. Sjöstrand et al, Comp. Phys. Comm., 135, 238 (2001).

7. J. Campbell et al, Phys Rev D, 67, 095002 (2003).

8. V. M. Abazov et al. (DØ Collaboration), Phys Rev D, 71, 072004 (2005).

9. V. M. Abazov et al. (DØ Collaboration), Phys. Rev. Lett., 95, 151801 (2005).

10. V. M. Abazov et al. (DØ Collaboration), Phys. Rev. Lett., 97, 121802 (2006).

11. A. Abulencia et al. (CDF Collaboration), Phys. Rev. Lett., 96, 011802 (2006). 